\title{
Real-time ultrasonography-magnetic resonance image fusion navigation for percutaneous transforaminal endoscopic discectomy
}

\author{
${ }^{*}$ Peigen Xie, PhD, ${ }^{1,4,5}$ Feng Feng, MD, ${ }^{1,4,5}$ Junyan Cao, MD, ${ }^{2,6}$ Zihao Chen, PhD, ${ }^{1,4,5}$ Bingjun He, PhD, ${ }^{3}$ \\ Zhuang Kang, PhD, ${ }^{3}$ Lei He, MD, ${ }^{1,4,5}$ Wenbin Wu, PhD, ${ }^{1,4,5}$ Lei Tan, MD, ${ }^{2,6} \mathrm{Kai}$ Li, PhD, ${ }^{2,6}$ \\ Rongqin Zheng, $\mathrm{PhD},{ }^{2,6}$ and Limin Rong, $\mathrm{PhD}^{1,4,5}$
}

\begin{abstract}
Departments of ${ }^{1}$ Spine Surgery, ${ }^{2}$ Ultrasound, and ${ }^{3}$ Radiology, The Third Affiliated Hospital of Sun Yat-sen University; ${ }^{4}$ Guangdong Provincial Center for Quality Control of Minimally Invasive Spine Surgery, The Third Affiliated Hospital of Sun Yat-sen University; ${ }^{5}$ Guangdong Provincial Center for Engineering and Technology Research of Minimally Invasive Spine Surgery; and ${ }^{6}$ Guangdong Key Laboratory of Liver Research, Guangzhou, China
\end{abstract}

OBJECTIVE Percutaneous transforaminal endoscopic discectomy (PTED) is usually performed under fluoroscopic guidance and is associated with a large radiation dose. Ultrasonography (US)-MR image fusion navigation combines the advantages of US and MRI and requires significantly less radiation than fluoroscopy. The purpose of this study was to evaluate the safety and effectiveness of US-MR image fusion navigation for PTED.

METHODS From January to September 2018, patients with L4-5 lumbar disc herniation requiring PTED were randomized to have the procedure conducted with US-MR image fusion navigation or fluoroscopy. The number of fluoroscopies, radiation dose, duration of imaging guidance, intraoperative visual analog scale (VAS) pain score, intraoperative complications, and clinical outcomes were compared between the groups.

RESULTS There were 10 patients in the US-MR navigation group and 10 in the fluoroscopy group, and there were no significant differences in age, sex ratio, or BMI between the 2 groups (all $p>0.05$ ). Intraoperatively, the total radiation dose, number of fluoroscopies performed, duration of image guidance, and VAS low-back and leg pain scores were all significantly lower in the US-MRI navigation group than in the fluoroscopy group (all $p<0.05$ ). There were no intraoperative complications in either group. Postoperative improvements in Japanese Orthopaedic Association, Oswestry Disability Index, and VAS pain scale scores were similar between the 2 groups.

CONCLUSIONS US-MR image fusion navigation is a promising technology for performing PTED and requires significantly less radiation than fluoroscopy.

Clinical trial registration no.: NCT03403244 (ClinicalTrials.gov).

https://thejns.org/doi/abs/10.3171/2020.1.SPINE191223

KEYWORDS image fusion; magnetic resonance; navigation; percutaneous transforaminal endoscopic discectomy; ultrasonography; lumbar

$\mathrm{L}$ UMBAR disc herniation (LDH) is the most common cause of sciatica, and the pain can severely affect work and quality of life. ${ }^{6}$ Percutaneous transforaminal endoscopic discectomy (PTED) has been demonstrated to be a safe and effective minimally invasive spine surgery for treating $\mathrm{LDH} .^{2}$ This procedure, however, is experience-dependent. The most important and difficult step is accurately inserting the puncture needle into the safe region at an appropriate puncture angle., ${ }^{9,10,12}$ Insertion of the puncture needle and dilator, as well as foraminoplasty, is performed using fluoroscopy or ultrasonography (US) guidance, or O-arm or CT navigation, with fluoroscopy the most commonly used method. The high-dose radiation exposure from fluoroscopy may have adverse effects on the skin, bone, parotid glands, and lungs. ${ }^{5,22}$ Compared with conventional C-arm navigation, $\mathrm{O}$-arm or $\mathrm{CT}$ navigation reduces the amount of radiation exposure to the surgeon, but does not significantly reduce the amount of

ABBREVIATIONS JOA = Japanese Orthopaedic Association; LDH = lumbar disc herniation; NTIP = needle tail intelligent positioning; ODI = Oswestry Disability Index; PTED = percutaneous transforaminal endoscopic discectomy; US = ultrasonography; VAS = visual analog scale.

SUBMITTED October 10, 2019. ACCEPTED January 2, 2020.

INCLUDE WHEN CITING Published online March 27, 2020; DOI: 10.3171/2020.1.SPINE191223.

${ }^{*}$ P.X. and F.F. contributed equally to this work. 
radiation exposure to the patient. In addition, these methods can only show bone structural landmarks; nerves and other important structures are not visualized. ${ }^{21}$ The use of US guidance avoids any radiation exposure; however, bone structures can impact US imaging and as a result the needle tip may not be visualized when it approaches the vertebrae. ${ }^{1}$ As such, real-time monitoring of the puncture needle is not possible, thus limiting the use of US guidance.

The US-MR image fusion navigation system combines the advantages of real-time US and high-resolution MRI. Studies reporting the use of this technique in liver ablation and prostate and brain surgeries have shown that it can accurately guide the puncture of targets that cannot be observed by US alone. ${ }^{13,17,18,20,23}$ A study has also shown the feasibility of real-time US-MR fusion imaging for lumbar facet joint injections. ${ }^{19}$

We hypothesized that US-MR image fusion navigation would provide real-time guidance, involve less radiation than fluoroscopy, and be accurate for performing intervertebral foramen puncture during PTED. However, there have been no reports examining the technique for PTED. Thus, the purpose of this study was to evaluate the feasibility, safety, and efficacy of performing PTED with USMR image fusion navigation.

\section{Methods}

Prior to the clinical study, a proof-of-concept animal study was performed with pigs to establish that US-MR image fusion navigation allowed correct placement of the needle and working cannula. The experiment showed that puncture under navigation with US-MR image fusion provided high accuracy for needle tip placement and effectively addressed the problem of difficulty visualizing the needle tip. Data from this experiment are on file and available upon request.

\section{Patients}

This prospective study was approved by the Clinical Trial Ethics Committee of our hospital and complied with the tenets of the Declaration of Helsinki. The trial is registered on ClinicalTrials.gov (no. NCT03403244). Written informed consent was obtained from all patients prior to their inclusion in the study.

The inclusion criterion was patients with L4-5 singlesegment LDH who required PTED. Patients who were not surgical candidates, who could not receive local anesthesia, and who declined participation in the study or could not commit to follow-up were excluded. Preoperatively, patients were randomized by lottery to receive PTED with US-MR image fusion navigation or G-/C-arm x-ray fluoroscopic guidance until there were 10 patients in each group.

\section{Surgical Procedure}

\section{Fluoroscopy Group}

A standard transforaminal endoscopic procedure was performed as described in the literature. ${ }^{2}$ Confirmation of the surgical segment, insertion of the needle and working cannula, and foraminoplasty with reamers were performed using C-/G-arm fluoroscopy.

\section{US-MR Image Fusion Navigation Group}

MRI was performed using a GE Discovery MR750w 3.0T device with a body coil (Fig. 1). The protocol included axial, sagittal, and coronal 3D FIESTA and LAVA flex sequences (TR $3.8 \mathrm{msec}$, TE minimum full, slice thickness $3.0 \mathrm{~mm}$, phase field of view 0.8 , flip angle $15^{\circ}$, slice gap $0 \mathrm{~mm}$ ). Before MRI examination, 5 external labels were placed on the back of the patient next to 5 vertebral spinous processes. The labels were positioned between the upper and lower vertebral bodies of the target segment, and within 5-10 cm from the midline of the spine. Labels were placed such that they were not on the same plane. The external labels were capsules containing a lipid-soluble vitamin solution that can be clearly visualized on an MR image. The scanning range was the lumbar vertebrae where the external labels were located. Prior to the surgical procedure, lumbar plexus MRI was performed to obtain enhanced images of the lumbar nerves. ${ }^{8}$

US was performed with a MyLab Twice US unit (Esaote) and linear array transducer LA523 (5-13 MHz). The US unit was equipped with software for automatic fusion of MRI sequences, the Virtual Navigator program, and needle tail intelligent positioning (NTIP).

For the procedure, the patient was placed prone and covered with a sterile sheet. An electromagnetic tracking system was placed at the front of the head. Acquired MRI sequences were inputted into the Esaote image fusion system. First, automatic fusion of MRI sequences from 3 directions was performed, after which the posterior-inferior edge of the intervertebral foramen outer aperture was labeled on the MR image. When performing preoperative MRI, 5-6 external labels adhered to the patient's back. These external labels showed a strong signal on the MRI. The external labels were then used for complete automatic fusion of US and MR images, and spinous processes were used for correct superimposition of US and MR images. During US-MR image registration, the MR image can be connected to the patient's body by determining the position of each external label. Automatic fusion of MRI sequences from 3 directions was performed, and then the posterior-inferior edge of the intervertebral foramen outer aperture was labeled on the MR image. The spinous processes were used for correct superimposition of US and MR images. The shape of the spinous process can be identified on the US image and compared with the MR image. When the two images were completely superimposed, the calibration of the fusion navigation was completed. The precision of this process was verified by applying the overlapping mode to show the spinous processes accurately superimposed on MR and US images. Then, US-MR image registration and fine-tuning were completed (Fig. 2). After US-MR image fusion, a total of 3 targets were identified and labeled: 1) the initial puncture target (at the outer aperture of the intervertebral foramen); 2) the endpoint target for implantation of the working cannula (at the inner aperture of the intervertebral foramen entrance); and 3) the nerve root protection target (Fig. 3).

Target puncture began after the NTIP device was installed and detected. The needle insertion process was based only on a virtual needle shown on the MR image. 

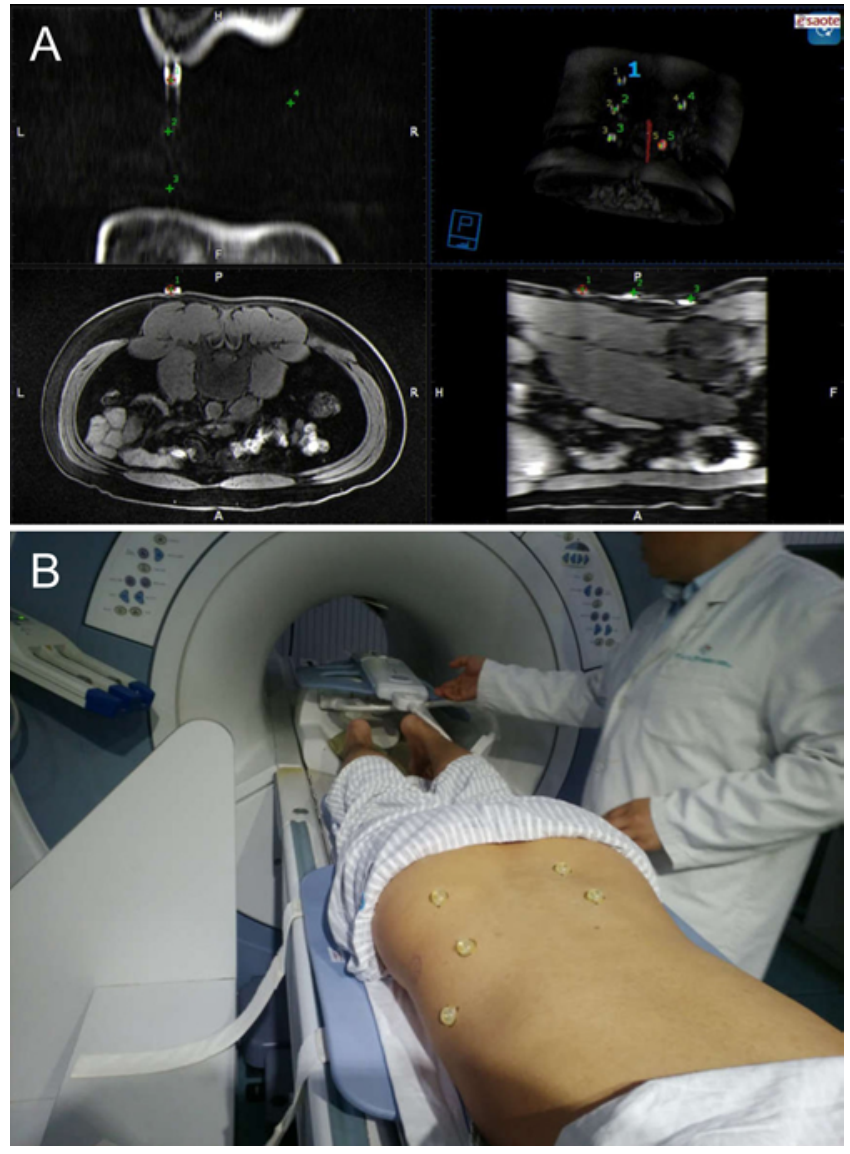

FIG. 1. A: MR images were downloaded and merged with the US images according to the labels affixed to the skin. B: During MRI examination patients were positioned prone, as they were for the surgical procedure. Figure is available in color online only.

Needle insertion was stopped when the MR image showed that the virtual needle tip had reached the center of the target. After infiltration of the subcutaneous tissue with local anesthesia, the puncture needle was selected and the NTIP positioning device was installed and detected. The previously labeled initial puncture target and endpoint target were identified. Nerve roots were also identified so as to be avoided. Needle puncture of the initial target was performed, and fluoroscopy was used to confirm that the puncture site was correct (Fig. 4). The NTIP device was then secured to the reamer and the dilator, and the intervertebral foramen was expanded. The reamer was guided to the endpoint with visualization on the navigation monitor. Fluoroscopy was used to confirm the reamer was correctly positioned and that the working cannula was established (Fig. 5). After construction of the cannula, endoscopic discectomy was performed as in the fluoroscopy group.

\section{Outcome Assessment}

Image fusion efficiency, the effectiveness of US-MR image guidance, and surgical clinical outcomes were assessed. Image fusion efficiency was defined as the time from when the MRI sequence images were imported into the system to when the US-MR image fusion was completed.

A number of measures were used to examine the effectiveness of US-MR image guidance. The number of fluoroscopies performed was summed from the beginning of surgery until the working cannula was successfully established. The total fluoroscopy radiation dose from the beginning of surgery to the establishment of the working cannula was calculated. The duration of image guidance was defined as the time from the beginning of surgery until the working cannula was established. As the procedure was performed under local anesthesia, patients were asked to rate intraoperative low-back and leg pain using a visual analog scale (VAS) during construction of the working cannula. After the intervertebral foramen was expanded, the working channel of the endoscope was placed. Prior to passage of the endoscope, the VAS assessment was performed, and the score was recorded by the assistant. Low-back and leg pain were rated together, with 0 indicating no pain and 10 indicating extreme pain. Finally, intraoperative complications including nerve root injury, CSF leakage, and inadvertent vessel and organ puncture were evaluated.

To evaluate surgical clinical outcomes, pre- and postoperative VAS low-back and leg pain score,,$^{15}$ Oswestry Disability Index (ODI), ${ }^{4}$ and Japanese Orthopaedic As-
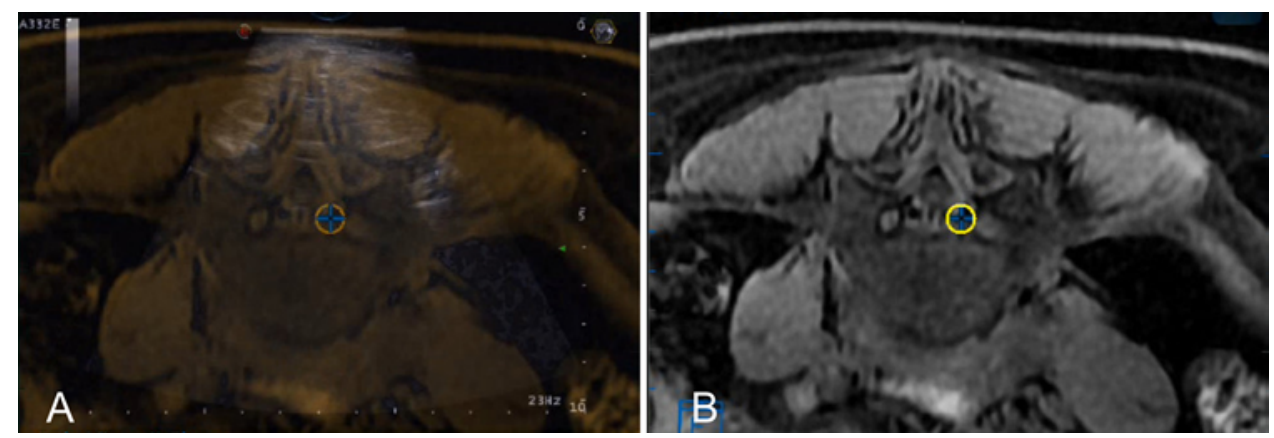

FIG. 2. The patient's position may shift slightly due to transfer and movement. Therefore, calibration was used to eliminate image deviation. The spinous processes (circles, blue crosses) and other bone landmarks can be easily identified on US images (A) and MR images (B). When the MRI scanning plane corresponds to the US scanning plane, calibration can be confirmed. Figure is available in color online only. 

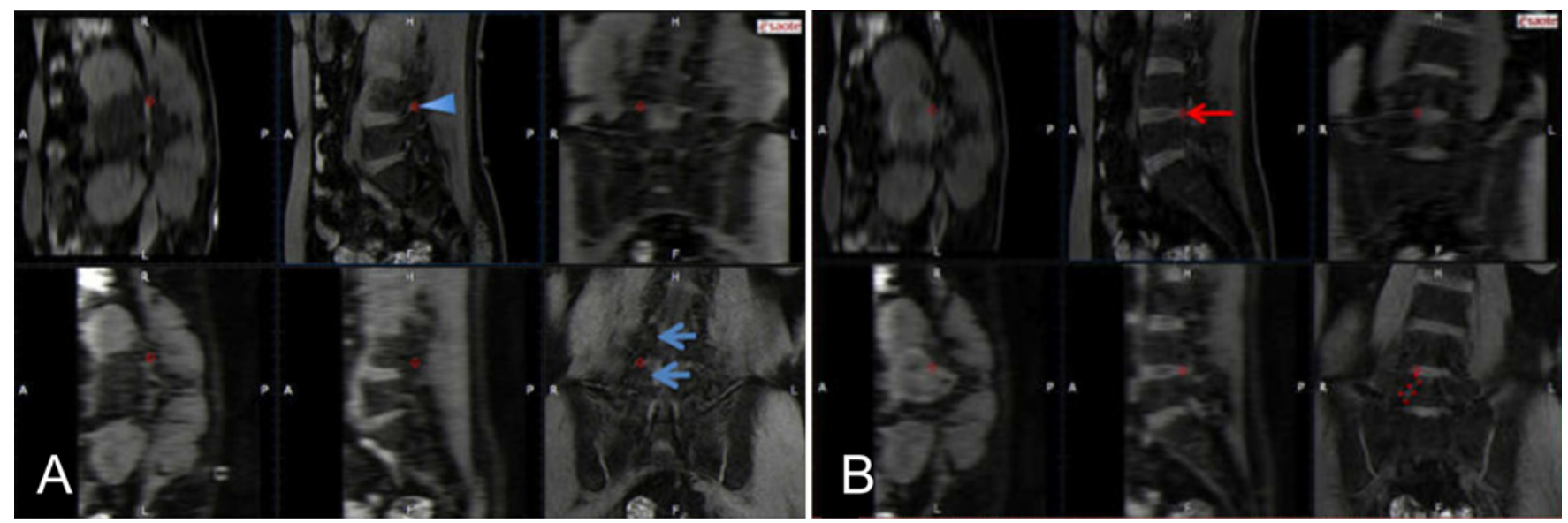

FIG. 3. A: The intervertebral foramen outer aperture, the initial puncture target (red dot), was identified and targeted by visualization of the affected upper articular process of L5 (blue arrowhead) on sagittal MR images, and the outer edge of the ipsilateral pedicle (blue arrows) on coronal images. B: The inner aperture of the L4-5 intervertebral foramen (red arrow) was the endpoint of the working passage (red dot) and was identified and targeted by visualization of the posterior margin of the L4-5 intervertebral disc and the posterior-superior angle of the L5 vertebral body on sagittal MR images, and the internal margin of the pedicle on coronal images. The L4-5 affected nerve root (red parallel dotted lines) was identified on the coronal plane and considered a protected target. In multiple MRI sequences, the nerve root and possible puncture target point can be easily identified and labeled with different colors. Figure is available in color online only.
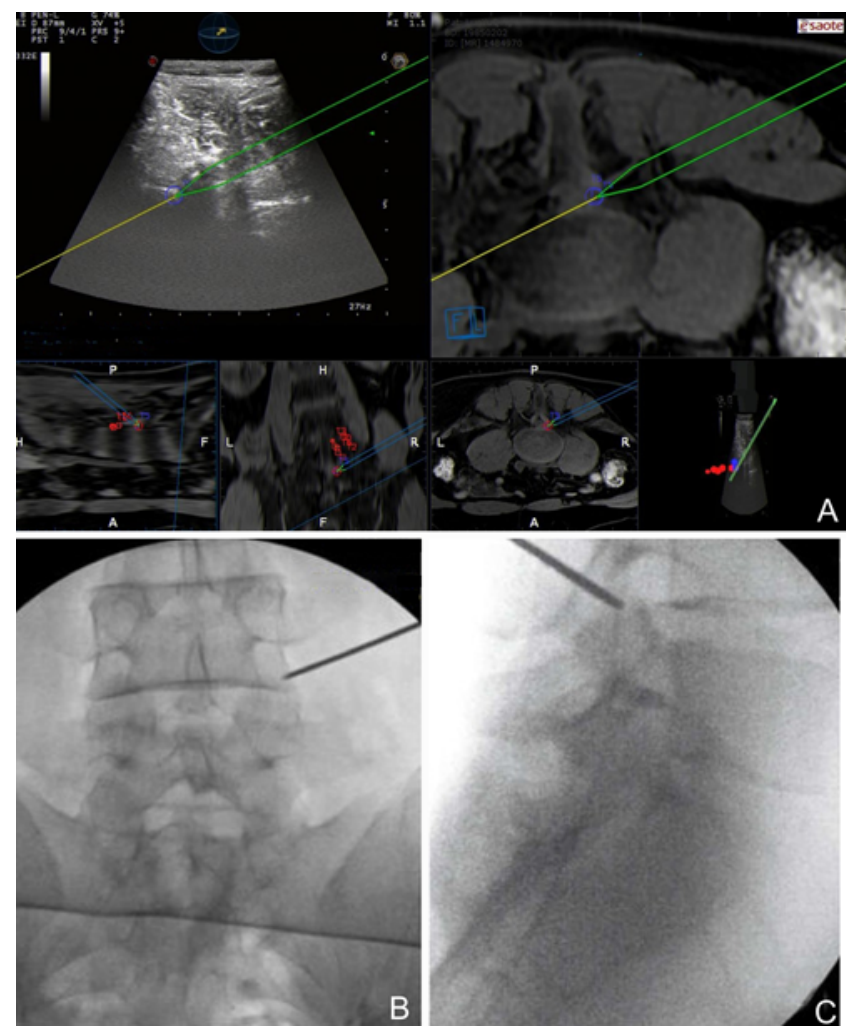

FIG. 4. A: The puncture needle tip is seen reaching the initial target (blue circle) guided by US-MR image fusion navigation. The nerve root and possible puncture target point were labeled with different colors and easily identified. The red dot is a nerve root, and the blue dot is the target point. B and C: Fluoroscopy confirmed that the needle tip was at the outer aperture of the intervertebral foramen. Figure is available in color online only. sociation (JOA) ${ }^{16}$ scale scores were evaluated. Outcomes were assessed by one of the authors (Z.C.).

\section{Statistical Analysis}

Statistical analyses were performed using SPSS software (version 16.0, SPSS Inc.). All measurements were expressed as mean values with 2 standard deviations, or the median. Differences between the US-MR navigation and fluoroscopy group were compared using the Student t-test and chi-square test. A p value $<0.05$ was considered statistically significant.

\section{Results}

From January to September 2018, 20 patients requiring PTED for an L4-5 LDH were enrolled in the study (Table 1). There were 10 patients in the US-MR image fusion navigation group and 10 in the fluoroscopy group, and there were no significant differences in age, sex ratio, or BMI between the 2 groups (all $\mathrm{p}>0.05$ ). The primary procedure was successfully completed in all patients in both groups with no conversions to open surgery. In the US-MR image fusion navigation group, the mean time required for prepuncture image fusion, registration, and target labeling was $32.6 \pm 7.2$ minutes.

Intraoperatively, the total radiation dose, number of fluoroscopies performed, duration of image guidance, and VAS low-back and leg pain scores were all significantly lower in the US-MRI navigation group than in the fluoroscopy group (all $\mathrm{p}<0.05$; Table 2 ). There were no intraoperative complications (such as nerve root injury, CSF leakage, or vessel or misplaced organ puncture) in either group.

Surgical outcomes are summarized in Table 3. Preoperative VAS pain, ODI, and JOA scores were similar between the 2 groups (all $p>0.05$ ). In both groups, the postoperative values of all 3 scores were significantly improved as compared to the preoperative values (all $\mathrm{p}<$ 

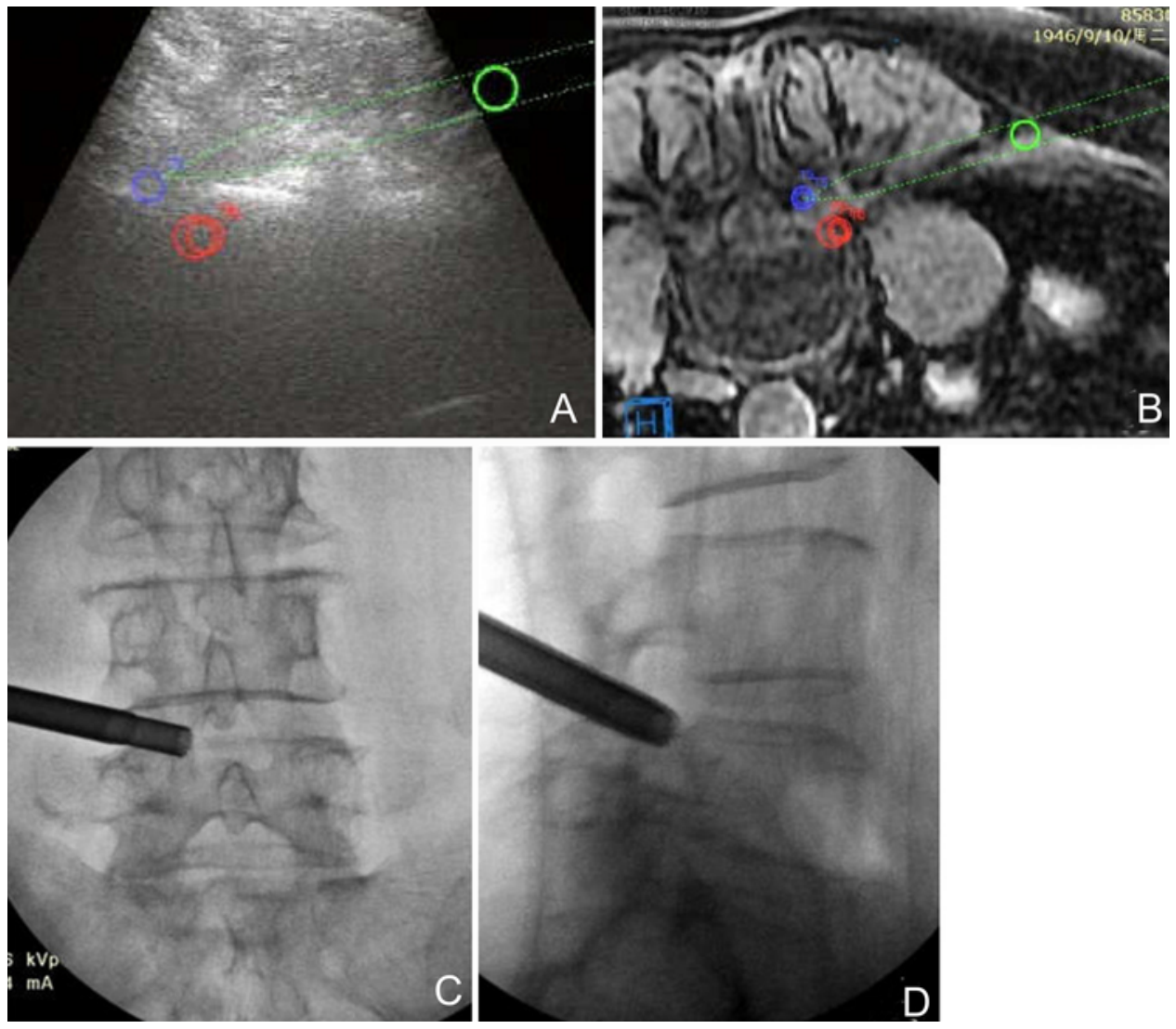

FIG. 5. A and B: Establishment of the working cannula by US-MR image fusion navigation. The reamer was guided to the endpoint (blue circle), and the nerve root (protected target; red circle) was avoided. The green circle indicates the section left by the working cannula in the ultrasonic plane. C and D: Fluoroscopy confirmed that the reamer was positioned correctly at the inner aperture of the intervertebral foramen entrance. Figure is available in color online only.

0.001). Furthermore, the postoperative values were similar between the 2 groups (all $\mathrm{p}<0.05$ ).

\section{Discussion}

The results of this study showed that US-MR image fusion navigation is accurate for performing PTED in patients with LDH and is associated with a significantly lower total radiation dose and less intraoperative pain than fluoroscopic guidance.

Klauser et al. ${ }^{11}$ first introduced US-CT image fusion navigation for sacroiliac joint injection and provided preliminary evidence of the utility of the technique. However, they noted that this technique could not show the location of needle tips inside joints. Subsequently, Sartoris et al. ${ }^{19}$ successfully performed lumbar facet joint injections under US-MR image fusion navigation. However, they claimed that the MR images of bone structures were not as good as the images obtained using CT. In both US-CT and US-MR image fusion navigation, the good spatial resolution of CT or MRI is combined with optimum real-time US. While there has been a slow but steady increase in the use of image fusion navigation for spine surgery in recent years, studies on PTED using US-MR image fusion navigation are rare.

Our results support the use of US-MR image fusion navigation for performing PTED. Importantly, MRI can clearly show the exit root. A previous study reported that the incidence of exit nerve root injury resulting in severe clinical symptoms during puncture and reamer grinding in PTED was $1.0 \%-8.9 \% .^{3}$ Labeling the nerve root during reaming can effectively prevent nerve injury. In this study, intraoperative low-back and leg pain VAS scores were lower in the US-MRI navigation group than in the fluoroscopy group, while no differences between the 2 groups for these scores were found postoperatively. Because navigation helps achieve more accurate puncture and avoids squeezing nerve roots during the expansion of the intervertebral foramen and channel establishment, it can reduce the pain during foraminoplasty.

MRI for bones is not as good as CT, and target labeling and navigation puncture usually require a combination of

\section{TABLE 1. Patient data}

\begin{tabular}{lccc}
\hline \multicolumn{1}{c}{ Variable } & $\begin{array}{c}\text { US-MR Navigation } \\
(\mathrm{n}=10)\end{array}$ & $\begin{array}{c}\text { Fluoroscopy } \\
(\mathrm{n}=10)\end{array}$ & $\begin{array}{c}\mathrm{p} \\
\text { Value }\end{array}$ \\
\hline Mean age $\pm \mathrm{SD}$, yrs & $39.5 \pm 17.2$ & $41 \pm 12.5$ & 0.543 \\
\hline Male, $\%$ & 70 & 80 & 0.606 \\
\hline Mean BMl $\pm \mathrm{SD}, \mathrm{kg} / \mathrm{m}^{2}$ & $24.18 \pm 4.62$ & $23.67 \pm 2.64$ & 0.208 \\
\hline
\end{tabular}


TABLE 2. Intraoperative outcome measures

\begin{tabular}{|c|c|c|c|}
\hline Intraop Outcome Measure & $\begin{array}{l}\text { US-MR } \\
\text { Navigation* }\end{array}$ & Fluoroscopy* & $\begin{array}{c}p \\
\text { Value }\end{array}$ \\
\hline Total radiation dose, mGy & $8.46 \pm 2.6$ & $28.3 \pm 5.1$ & $<0.001$ \\
\hline No. of fluoroscopies & $11.0 \pm 3.1$ & $32.6 \pm 4.5$ & $<0.001$ \\
\hline $\begin{array}{l}\text { Duration of image guidance, } \\
\text { mins } \dagger\end{array}$ & $20.5 \pm 3.6$ & $23.4 \pm 1.9$ & 0.039 \\
\hline $\begin{array}{l}\text { Intraop low-back and leg VAS } \\
\text { pain score }\end{array}$ & $3.6 \pm 0.8$ & $4.8 \pm 1.0$ & 0.011 \\
\hline
\end{tabular}

${ }^{*}$ Mean \pm SD.

$\dagger$ Time from beginning of operation to establishment of working cannula.

sequence reconstructions with blurred images from other positions for identifying the bone structures. To address this problem, we simultaneously obtained vertebral MRI sequences from multiple positions and performed automatic fusion before US-MR image fusion to avoid the use of blurry MR images from other reconstruction sites. This method is different from that used by Sartoris et al., ${ }^{19}$ in which the vertebrae sequence from a single position was used. The MR images were obtained before surgery. Due to changes in body position during surgery, "navigation drift" may occur. Therefore, it is necessary to perform US calibration before starting the puncture. The spinous processes and other bone landmarks can be used to fine-tune the alignment until the US-MR images are completely superimposed. If the patient's position changes during surgery, it is also recommended to perform US calibration again to ensure the accuracy of navigation.

Another important part of our method is that NTIP was used to indicate the real-time position of the needle tip. This allows for complete visualization of the needle tip during insertion, and highly accurate placement. Our results showed that US-MR image fusion navigation provided comparable placement accuracy with fluoroscopy, but with a significantly lower radiation dose, no significant complications, and similar clinical outcomes. While USMR image fusion navigation still requires fluoroscopy to confirm puncture and cannula sites, we believe once a surgeon has become very proficient with the technique, fluoroscopic confirmations will not be necessary and a "zero radiation" procedure can be achieved.

In addition, the preclinical animal experiment showed that while an operator who was inexperienced with the technique took longer to perform the procedure than an experienced operator, outcomes including accurate needle tip placement, puncture errors, and operating errors were not different (data on file).

In a recent study, Liu et al. ${ }^{14}$ studied volume navigation with fusion of real-time US and CT images to guide puncture in PTED. They found that puncture time and frequency of fluoroscopy were significantly lower when volume navigation was used as compared to C-arm fluoroscopy, and that ODI and VAS pain scores were similar between both groups of patients. While using CT reduces the amount of intraoperative radiation (compared to fluoroscopy), preoperative radiation is not reduced, whereas it is eliminated with MRI. MRI also provides better visualization of blood vessels and nerves, and thus can theoreti-
TABLE 3. Clinical outcome measures

\begin{tabular}{lccc}
\hline Outcome Measure & US-MR Navigation & Fluoroscopy & $p$ Value \\
\hline Preop VAS pain score & $6.5 \pm 0.8$ & $7.2 \pm 1.0$ & 0.115 \\
\hline Postop VAS pain score & $1.0 \pm 0.7$ & $1.1 \pm 0.6$ & 0.729 \\
\hline Intragroup p value & $<0.001$ & $<0.001$ & \\
\hline Preop ODI & $57.8 \pm 10.3$ & $62.6 \pm 7.0$ & 0.237 \\
\hline Postop ODI & $14.2 \pm 5.4$ & $12.2 \pm 2.0$ & 0.29 \\
\hline Intragroup p value & $<0.001$ & $<0.001$ & \\
\hline Preop JOA score & $17.5 \pm 2.9$ & $15.5 \pm 2.1$ & 0.091 \\
\hline Postop JOA score & $25.7 \pm 0.9$ & $25.5 \pm 0.5$ & 0.567 \\
\hline Intragroup p value & $<0.001$ & $<0.001$ & \\
\hline
\end{tabular}

cally reduce the risk of intraoperative injury to a greater degree than CT. In addition, as compared to the study of Lui et al., our study used US-MR image fusion navigation for enlargement of the intervertebral foramen and cannula placement, as well as the puncture process. Because most nerve root damage occurs during enlargement of the intervertebral foramen and cannula placement, MRI is more advantageous than CT in avoiding nerve injury.

The main disadvantage of our US-MR image fusion navigation system is that our MR images were obtained preoperatively. During intraoperative US-MR image fusion, patient position and the locations of the external calibration labels may be different from those preoperatively, and this can adversely affect the accuracy of image fusion. Hakime et al. ${ }^{7}$ reported that CT or MRI performed immediately or shortly before surgery provides significantly higher accuracy of US fusion images than if CT or MRI is performed earlier in advance of the procedure. Because the MRI data were collected before the operation, the accuracy of navigation would be decreased due to the change in body position or the movement of markers. Therefore, the interval between MRI examination and operative time should be as short as possible. As such, we attempted to shorten the interval between the preoperative MRI examination and the surgery to improve the accuracy of image fusion.

According to our current experience, a doctor can become proficient in the US-MRI navigational PTED after only 3-5 practice cases. Navigation error can be less than $2 \mathrm{~mm}$. The percutaneous endoscopy usually requires multiple fluoroscopic images, with an average of 30-40. Although the price is inexpensive, tube endoscopy or microsurgery causes more damage to dorsal muscle column structures. In China, the costs of microendoscopy and spinal percutaneous endoscopy are not that different, i.e., \$2000 and \$3000 (in United States dollars), respectively. Even with ultrasound navigation, only $\$ 200$ is added to the cost. Compared to conventional endoscopy, ultrasound navigation endoscopy is safe, portable, and inexpensive. More studies should be conducted to investigate the costeffectiveness in the future.

There are limitations to our study that should be considered. First, our study was not a randomized controlled trial as the sample size was not precalculated according to the scale of the study, but set to 10 patients per group. Although the 2 groups of patients had good comparability, randomization errors can be reduced to a greater extent 
if randomization and blinding allocation are conducted, which will improve the study quality. The number of patients was small, which affects the statistical power of the results. Fluoroscopy was still required with US-MRI navigation, although the radiation dose was significantly lower. We hope to eliminate the need for fluoroscopy with further refinement of the technique.

\section{Conclusions}

US-MR image fusion navigation provides high-resolution visualization of anatomical structures and real-time monitoring of needle placement during PTED. The technique provides similar clinical outcomes to fluoroscopic guidance, with significantly less intraoperative radiation and pain.

\section{Acknowledgments}

This study was supported by the National Natural Science Foundation of China (grant no. 81430038) and the Medical Scientific Research Foundation of Guangdong Province, China (grant no. A2017006).

\section{References}

1. Abu-Zidan FM, Hefny AF, Corr P: Clinical ultrasound physics. J Emerg Trauma Shock 4:501-503, 2011

2. Chen Z, Zhang L, Dong J, Xie P, Liu B, Wang Q, et al: Percutaneous transforaminal endoscopic discectomy compared with microendoscopic discectomy for lumbar disc herniation: 1-year results of an ongoing randomized controlled trial. J Neurosurg Spine 28:300-310, 2018

3. Choi I, Ahn JO, So WS, Lee SJ, Choi IJ, Kim H: Exiting root injury in transforaminal endoscopic discectomy: preoperative image considerations for safety. Eur Spine J 22:2481-2487, 2013

4. Fairbank JC, Pynsent PB: The Oswestry Disability Index. Spine (Phila Pa 1976) 25:2940-2952, 2000

5. Falavigna A, Ramos MB, Iutaka AS, Menezes CM, Emmerich J, Taboada N, et al: Knowledge and attitude regarding radiation exposure among spine surgeons in Latin America. World Neurosurg 112:e823-e829, 2018

6. Gibson JN, Waddell G: Surgical interventions for lumbar disc prolapse: updated Cochrane Review. Spine (Phila Pa 1976) 32:1735-1747, 2007

7. Hakime A, Deschamps F, De Carvalho EG, Teriitehau C, Auperin A, De Baere T: Clinical evaluation of spatial accuracy of a fusion imaging technique combining previously acquired computed tomography and real-time ultrasound for imaging of liver metastases. Cardiovasc Intervent Radiol 34:338-344, 2011

8. He L, Kang Z, Tang WJ, Rong LM: A MRI study of lumbar plexus with respect to the lateral transpsoas approach to the lumbar spine. Eur Spine J 24:2538-2545, 2015

9. Hsu HT, Chang SJ, Yang SS, Chai CL: Learning curve of fullendoscopic lumbar discectomy. Eur Spine J 22:727-733, 2013

10. Iprenburg M, Wagner R, Godschalx A, Telfeian AE: Patient radiation exposure during transforaminal lumbar endoscopic spine surgery: a prospective study. Neurosurg Focus 40(2):E7, 2016

11. Klauser AS, De Zordo T, Feuchtner GM, Djedovic G, Weiler RB, Faschingbauer R, et al: Fusion of real-time US with CT images to guide sacroiliac joint injection in vitro and in vivo. Radiology 256:547-553, 2010

12. Lee DY, Lee SH: Learning curve for percutaneous endoscopic lumbar discectomy. Neurol Med Chir (Tokyo) 48:383-389, 2008
13. Li K, Su ZZ, Xu EJ, Ju JX, Meng XC, Zheng RQ: Improvement of ablative margins by the intraoperative use of CEUS$\mathrm{CT} / \mathrm{MR}$ image fusion in hepatocellular carcinoma. BMC Cancer 16:277, 2016

14. Liu YB, Wang Y, Chen ZQ, Li J, Chen W, Wang CF, et al: Volume navigation with fusion of real-time ultrasound and CT images to guide posterolateral transforaminal puncture in percutaneous endoscopic lumbar discectomy. Pain Physician 21:E265-E278, 2018

15. Mills RJ, Renfrew S: Measurement of pain threshold by thermal contact. Lancet 1:738-739, 1971

16. Ohtori S, Ito T, Yamashita M, Murata Y, Morinaga T, Hirayama J, et al: Evaluation of low back pain using the Japanese Orthopaedic Association Back Pain Evaluation Questionnaire for lumbar spinal disease in a multicenter study: differences in scores based on age, sex, and type of disease. J Orthop Sci 15:86-91, 2010

17. Prada F, Del Bene M, Mattei L, Lodigiani L, DeBeni S, Kolev V, et al: Preoperative magnetic resonance and intraoperative ultrasound fusion imaging for real-time neuronavigation in brain tumor surgery. Ultraschall Med 36:174-186, 2015

18. Puech $\mathrm{P}$, Rouvière $\mathrm{O}$, Renard-Penna R, Villers A, Devos $\mathrm{P}$, Colombel M, et al: Prostate cancer diagnosis: multiparametric MR-targeted biopsy with cognitive and transrectal USMR fusion guidance versus systematic biopsy-prospective multicenter study. Radiology 268:461-469, 2013

19. Sartoris R, Orlandi D, Corazza A, Sconfienza LM, Arcidiacono A, Bernardi SP, et al: In vivo feasibility of real-time MR-US fusion imaging lumbar facet joint injections. J Ultrasound 20:23-31, 2017

20. Siddiqui MM, George AK, Rubin R, Rais-Bahrami S, Parnes HL, Merino MJ, et al: Efficiency of prostate cancer diagnosis by MR/ultrasound fusion-guided biopsy vs standard extended-sextant biopsy for MR-visible lesions. J Natl Cancer Inst 108:djw039, 2016

21. Theologis AA, Burch S: Safety and efficacy of reconstruction of complex cervical spine pathology using pedicle screws inserted with stealth navigation and 3D image-guided (O-Arm) technology. Spine (Phila Pa 1976) 40:1397-1406, 2015

22. Wagner LK, Eifel PJ, Geise RA: Potential biological effects following high X-ray dose interventional procedures. J Vasc Interv Radiol 5:71-84, 1994

23. Zhong-Zhen S, Kai L, Rong-Qin Z, Er-Jiao X, Ting Z, AoHua Z, et al: A feasibility study for determining ablative margin with 3D-CEUS-CT/MR image fusion after radiofrequency ablation of hepatocellular carcinoma. Ultraschall Med 33:E250-E255, 2012

\section{Disclosures}

The authors report no conflict of interest concerning the materials or methods used in this study or the findings specified in this paper.

\section{Author Contributions}

Conception and design: Rong, Li, Zheng. Acquisition of data: Chen, B He, Kang, L He, Wu. Analysis and interpretation of data: Xie, Feng, Cao. Drafting the article: Xie, Feng, Cao. Critically revising the article: Xie, Feng. Reviewed submitted version of manuscript: Rong, Xie, Feng, Chen, B He, Wu. Approved the final version of the manuscript on behalf of all authors: Rong. Statistical analysis: Cao. Administrative/technical/material support: Cao, Kang, L He, Tan. Study supervision: Rong, Li, Zheng.

\section{Correspondence}

Limin Rong: The Third Affiliated Hospital of Sun Yat-sen University, Guangzhou, China. ronglm21@163.com. 